\title{
Annotations
}

\section{Interior spaces, northern places: Nunavut artist Annie Pootoogook's artwork is observational and narrative}

$\mathrm{U}$ nflinching, she depicts modern Inuit life as she sees it, rather than producing the traditional scenes that people in southern Canada have come to expect. There is tradition in Pootoogook's work, however. It is the Inuit concept of sulijuk, meaning when something "is true or real." Her focus is on scenes of prosaic domestic tranquility as well as domestic abuse - her own and that of other Inuit women mental illness and alcohol abuse.

Living in Cape Dorset, Baffin Island, Pootoogook is described as a chronicler. Pootoogook, herself, comments that her work is based on "emotional" or "difficult" incidents that she has seen and she wants to "show that [in her art]." Each drawing, she adds, "depends on how she is feeling" and "if there has been a good or bad time." Her artwork is a safe venue for her emotions, her interior spaces, to be expressed. Realized with coloured pencil on paper, her stark images reveal Inuit life as it adapts to accommodate southern culture within a still relatively closed society.

Born in 1969 , Pootoogook began drawing in 1997 , with the encouragement of the West Baffin Eskimo Cooperative Limited in Cape Dorset. She is represented by Feheley Fine Arts, Toronto. Her first public exhibit was at The Power Plant Contemporary Art Gallery, Toronto.

Pootoogook is on the national short list for 2006 Sobey Art Award. This biannual award is the pre-eminent award for Canadian artists aged 39 years or younger, the winner to be announced Nov. 7, 2006.

An exhibition at the Musée des beaux-arts de Montréal, in Montréal, featuring the works of Pootoogook and the four other Award finalists, will run Oct. 25, 2006, to Jan. 7, 2007.

\section{J. Lynn Fraser}

Freelance writer

Ottawa, Ont.

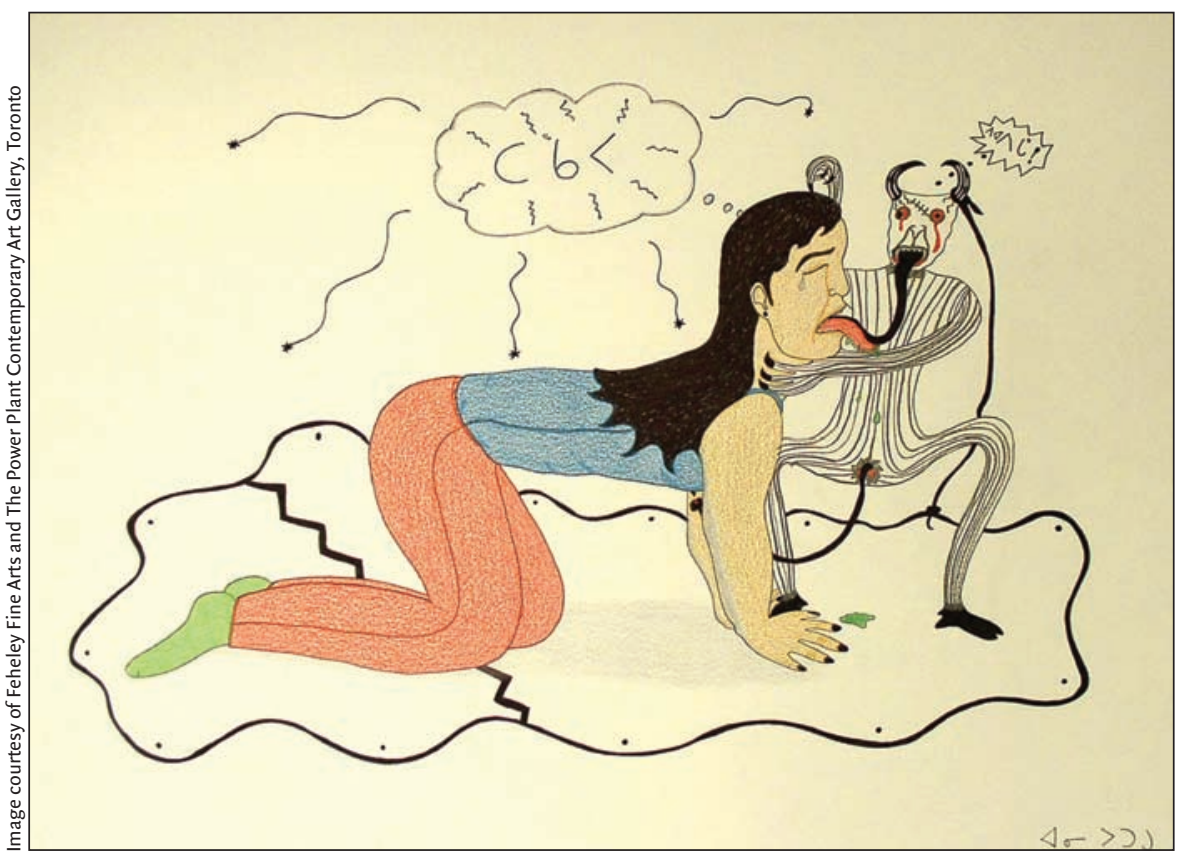

Annie Pootoogook, Woman with the Devil (2003/04). Pencil crayon, ink. $50.8 \times 66 \mathrm{~cm}$. Collection of Jay Smith \& Laura Rapp, Toronto.

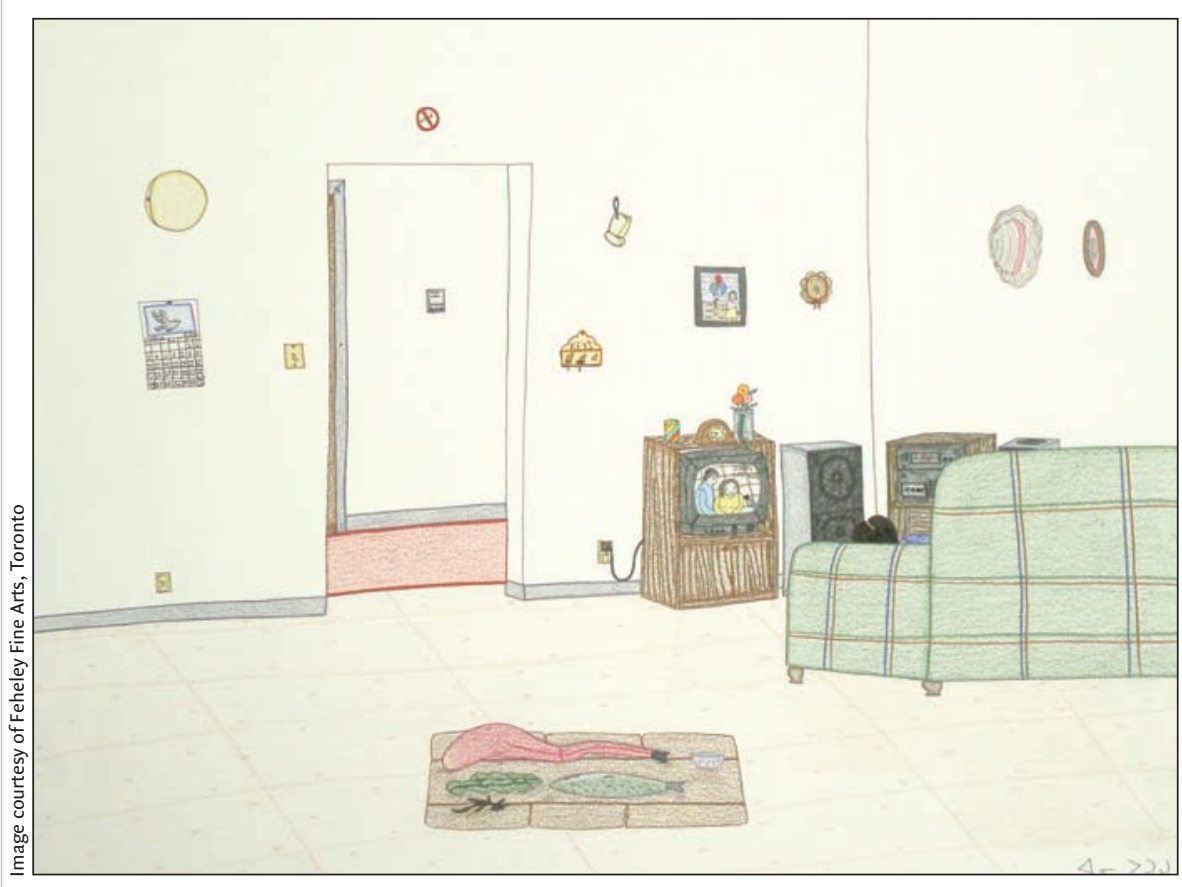

Annie Pootoogook, Watching Jerry Springer (2002/03). Pencil crayon and ink on paper. $20 \times 26$ in. 